\title{
EFEITOS DE EXERCÍCIOS FÍSICOS SOBRE FATORES DE RISCO CARDIOVASCULAR EM IDOSOS HIPERTENSOS
}

\section{Effects of physical exercise on cardiovascular risk factors in hypertensive older adults \\ Efectos de los ejercicios físicos en los factores de riesgo cardiovascular de mayores hipertensos}

\author{
Marinella Nogueira da Silva Hortencio \\ Universidade de Santo Amaro - UNISA - São Paulo (SP) - Brasil
}

Joycy Kelle Souza da Silva

Universidade de Santo Amaro - UNISA - São Paulo (SP) - Brasil

Marco Antonio Zonta

Universidade de Santo Amaro - UNISA - São Paulo (SP) - Brasil

Carlos Pereira Araújo de Melo

Universidade de Santo Amaro - UNISA - São Paulo (SP) - Brasil

\section{Carolina Nunes França}

Universidade de Santo Amaro - UNISA - São Paulo (SP) - Brasil

Universidade Federal de São Paulo - UNIFESP - São Paulo (SP) - Brasil

\section{RESUMO}

Objetivo: Avaliar os efeitos de um programa de exercícios na redução dos fatores de risco cardiovascular em idosos sedentários e hipertensos. Métodos: Trata-se de ensaio clínico não randomizado, realizado em uma clínica escola de fisioterapia em São Paulo, em 2016, com amostra de 34 idosos que realizaram um programa de exercícios de noventa minutos, duas vezes por semana, durante o período de três meses. As variáveis avaliadas foram: pressão arterial, índice de massa corporal, frações de colesterol, colesterol total e triglicérides. Houve comparação dos dados em dois momentos, antes do início e ao término do programa de exercícios, considerando-se $\mathrm{p}<0,0001$ em todos os casos pelo teste de Wilcoxon. Resultados: Encontrou-se idade mediana dos avaliados (intervalo interquartis) de 63 (61-66) anos, sendo 25 (74\%) do sexo feminino. Após três meses, houve redução estatisticamente significativa do peso, índice de massa corpórea, pressão arterial sistólica (PAS) e diastólica (PAD) dos idosos avaliados ( $<$ 0,0001 em todos os casos). Conclusão: O efeito do programa de exercícios foi eficaz na redução dos fatores de risco cardiovascular dos idosos sedentários e hipertensos estudados.

Descritores: Doenças Cardiovasculares; Hipertensão Arterial; Exercício; Idoso.

Registro Brasileiro de Ensaios Clínicos: RBR-4nn8j5

\section{ABSTRACT}

Objective: To assess the effects of an exercise program on the reduction of cardiovascular risk factors in sedentary hypertensive older adults. Methods: This is a nonrandomized clinical trial carried out in a physical therapy teaching clinic in São Paulo in 2016 with a sample of 34 older adults who performed a ninety-minute exercise program twice a week for three months. The analyzed variables were: blood pressure, body mass index, cholesterol fractions, total cholesterol and triglycerides. Data were compared in two stages, before and after the exercise program, with $p<0.0001$ in all cases using the Wilcoxon test. Results: The median age of the participants (interquartile range) was 63 (6166) years, with 25 (74\%) women. After three months, there was a statistically significant reduction in weight, body mass index, systolic (SBP) and diastolic blood pressure $(D B P)$ in the analyzed older adults $(p<0.0001$ in all cases). Conclusion: The exercise program was effective in reducing cardiovascular risk factors in the sedentary hypertensive older adults analyzed.

Descriptors: Cardiovascular diseases; Hypertension; Exercise; Aged.

Brazilian Clinical Trials Registry: RBR-4nn8j5 


\section{RESUMEN}

Objetivo: Evaluar los efectos de un programa de ejercicios para la disminución de los factores de riesgo cardiovascular de mayores sedentarios e hipertensos. Métodos: Se trata de un ensayo clínico no randomizado realizado en una clínica escuela de fisioterapia de São Paulo, en 2016, con una muestra de 34 mayores que realizaron un programa de ejercicios de noventa minutos, dos veces a la semana durante el periodo de tres meses. Las variables evaluadas fueron: la presión arterial, el índice de masa corporal, las fracciones de colesterol, el colesterol total y los triglicéridos. La comparación de los datos se dio en dos momentos: antes del inicio y al final del programa de ejercicios con p<0,0001 para todos los casos a través del test de Wilcoxon. Resultados: La edad mediana de los evaluados (intervalo intercuartil) fue de 63 (61-66) años y 25 (74\%) años para el sexo femenino. Tras tres meses hubo reducción estadísticamente significativa del peso, el indice de masa corporal y la presión arterial sistólica (PAS) y diastólica (PAD) de los mayores evaluados ( $p<0,0001$ para todos los casos). Conclusión: El efecto del programa de ejercicios ha sido eficaz para la reducción de los factores de riesgo cardiovascular de los mayores sedentarios e hipertensos estudiados.

Descriptores: Enfermedades Cardiovasculares; Hipertensión; Ejercicio; Anciano.

\section{INTRODUÇÃO}

As doenças cardiovasculares (DCV) fazem parte de um grupo chamado de doenças crônicas não transmissíveis (DCNT), sendo mais comuns as isquêmicas do coração, acidente vascular encefálico (AVE) e doença vascular periférica (DVP) ${ }^{(1-3)}$. Hoje, representam uma ameaça à saúde, sendo alvo constante das políticas de saúde pública no mundo devido às morbidades associadas. Causam redução significativa da produtividade, incapacidades, efeitos adversos na qualidade de vida e custos materiais diretos aos pacientes e familiares, além de um importante impacto financeiro sobre o sistema de saúde $\mathrm{d}^{(1,3)}$.

Os programas de exercícios físicos representam uma importante estratégia na prevenção e tratamento das $\mathrm{DCV}{ }^{(2,4)}$. Tratam-se de terapêuticas não farmacológicas que agem diretamente nos fatores de risco associados, como hipertensão arterial sistêmica (HAS), obesidade, dislipidemia e diabetes mellitus $(\mathrm{DM})^{(4)}$. A prática regular de exercícios pode reduzir significativamente $\mathrm{o}$ risco de mortalidade cardiovascular em até $60 \%$. No entanto, a prescrição deve levar em conta a avaliação do risco cardiovascular global, as condições sociais e interesses do paciente, a fim de promover a boa aderência às mudanças no estilo de vida ${ }^{(5)}$.

A HAS é considerada um importante fator de risco para ocorrência de eventos cardíacos. É uma condição clínica multifatorial caracterizada por níveis elevados e sustentados de pressão arterial (PA), podendo causar lesões em órgãos vitais, como cérebro, coração e rins, e de alta prevalência em todos os países, sejam desenvolvidos, sejam em desenvolvimento ${ }^{(2,5,5)}$. Estima-se que exista um bilhão de pessoas hipertensas no mundo: só nos EUA, entre 24 a 29\% de adultos economicamente ativos; enquanto, no Brasil, a estimativa é de 22 a 44\%. A HAS tem sido um dos maiores problemas de saúde pública da atualidade por estar diretamente relacionada às $\mathrm{DCV}$ e seus agravos ${ }^{(1,5)}$.

Destaca-se o importante efeito hipotensor dos exercícios aeróbios na proteção contra eventos cardiovasculares ${ }^{(7)}$. Uma redução de apenas $3 \mathrm{mmHg}$ para a pressão arterial sistólica (PAS) pode significar diminuição de 5-9\% e 8-14\% para risco cardiovascular e de infarto agudo do miocárdio (IAM), respectivamente. Esse efeito pode ser observado após exercícios aeróbios de baixa, moderada e elevada intensidades, porém só tem valor significativo se permanecer ao longo das 24 horas subsequentes. Uma única sessão de exercícios aeróbios produz uma hipotensão pós-exercício associada a uma sustentada vasodilatação ${ }^{(8)}$. Dessa forma, mostra-se ainda mais relevante a prática por indivíduos pré-hipertensos.

De acordo com a I Diretriz de Prevenção Cardiovascular( ${ }^{(7)}$, recomenda-se pelo menos 30 minutos por dia, cinco vezes por semana, de exercício moderado, para indivíduos idosos ou não, com ou sem HAS. Para idosos inativos, pode ser difícil tornar-se ativo e cumprir a recomendação atual, sendo a falta de motivação a principal questão envolvida.

O processo de envelhecimento tem recebido grande atenção mundial e ações são direcionadas aos idosos, estimulando a prática de exercícios, para assim reduzir os efeitos das doenças crônico-degenerativas, permitindo maior qualidade de vida ${ }^{(9,10)}$. Os programas sociais que estão destinados a essa população ofertam principalmente atividades aeróbias, como caminhadas ou hidroginástica, e são frequentemente recomendadas como uma forma importante para amenizar disfunções como a $\mathrm{HAS}^{(10)}$. Exercícios aeróbicos, circuitos, exercícios aquáticos, treinos de flexibilidade e técnicas de relaxamento são métodos não farmacológicos utilizados para controlar os fatores de risco cardiovascular ${ }^{(11,12)} \mathrm{em}$ idosos, com excesso de peso ou não ${ }^{(13)}$. Em hipertensos idosos, a prescrição adequada dos exercícios (em relação ao tipo, intensidade, duração e intervalos de recuperação) pode contribuir para incentivar, motivar e promover a adesão dos pacientes ${ }^{(9,11)}$.

A HAS associa-se ao aumento da adiposidade abdominal, sendo responsável por 65 a $75 \%$ dos casos de hipertensão primária $^{(12)}$. Cronicamente, esses estados levam a maiores agravos, como o desenvolvimento de HAS de difícil tratamento, resistência à insulina, DM, e lesões de órgãos alvo, como insuficiência renal (IR), DVP, maiores chances de AVE e IAM ${ }^{(3-5)}$, exigindo maiores gastos do sistema de saúde ${ }^{(12,13)}$, e frequentemente necessitando do uso de múltiplas drogas e do tratamento de outros fatores de risco, incluindo dislipidemia ${ }^{(12)}$. 
Dessa forma, os programas de incentivo à prática de exercícios físicos são um importante recurso no combate ao sobrepeso e à adiposidade abdominal, promovendo aumento do gasto energético, tanto agudamente, durante a sua realização, quanto cronicamente, estando relacionado à alterações da taxa metabólica de repouso (TMR) devido a modificações na composição corporal $^{(14)}$.

Entre os fatores de risco que provocam o desenvolvimento das DCV, também se encontram as dislipidemias, que são distúrbios do metabolismo lipídico, com repercussões sobre os níveis das lipoproteínas na circulação sanguínea. Altos níveis de triglicerídeos (TG) e de lipoproteína de baixa densidade (LDL-Colesterol), associados a baixos níveis de lipoproteínas de alta densidade (HDL-Colesterol) no sangue, costumam estar relacionados com a obesidade abdominal ${ }^{(15)}$.

As doenças cardiovasculares e os fatores de risco associados constituem um problema de saúde pública, sendo necessária a implantação de ações de promoção da saúde ${ }^{(1,3)}$. Trata-se de uma maneira de capacitação da comunidade e maior participação no processo de melhoria da sua qualidade de vida, descrita pela Organização Mundial de Saúde (OMS), como um estado de completo bem-estar físico, mental e social, enfatizando aspectos pessoais bem como as capacidades físicas, e não só a ausência de doença $\mathrm{a}^{(16,17) \text {. }}$

Nesse sentido, o objetivo do presente trabalho foi avaliar os efeitos de um programa de exercícios na redução dos fatores de risco cardiovascular em idosos sedentários e hipertensos.

\section{MÉTODOS}

Trata-se de um ensaio clínico com uma amostra de 34 pacientes idosos, previamente sedentários há pelo menos um ano, hipertensos em uso de medicação anti-hipertensiva, e liberados por médico para a prática de exercícios físicos leves a moderados.

O conceito de idoso foi definido a partir da idade cronológica do indivíduo, ou seja, considerando-se idosa a pessoa com 60 anos ou mais em países em desenvolvimento ${ }^{(18,19)}$. Cabe ressaltar que não houve grupo controle nem randomização neste estudo, incluindo-se todos os pacientes que frequentavam a Clínica Escola de Fisioterapia da Universidade Santo Amaro e que concordaram com sua participação no estudo.

A seleção dos pacientes ocorreu durante o mês de março de 2016, utilizando-se listagem do banco de dados eletrônico da Clínica Escola de Fisioterapia da Universidade Santo Amaro Campus I, localizada no município de São Paulo, São Paulo, Brasil, sendo o diagnóstico inicial de hipertensão arterial sistêmica, determinado por meio de dados do prontuário médico dos pacientes. Aqueles idosos que mostraram interesse em participar e que estavam de acordo com os critérios de inclusão nesta pesquisa compareceram ao Campus I da UNISA para assinar o Termo de Consentimento Livre e Esclarecido (TCLE). Os pacientes passaram por consulta com médica colaboradora deste estudo, e aqueles que receberam liberação foram atendidos pela fisioterapeuta responsável pelo estudo para a coleta de dados antropométricos e aferição da pressão arterial em repouso.

Inicialmente, selecionaram-se 54 pacientes; todos informados de que o controle de presença seria feito por lista de chamada e que aqueles que excedessem o número de faltas seriam automaticamente excluídos do programa, sendo o total de ausências permitidas de, no máximo, duas por mês, ou seja, total de 25\%. Do total de 54 pacientes selecionados no início deste protocolo, 20 não completaram o programa por problemas pessoais ou por terem excedido o limite de ausências; assim, participaram do estudo 34 idosos.

As aferições da pressão arterial ocorreram com esfignomanômetro digital, aprovado pelo INMETRO, com o braço apoiado sobre uma mesa, estando os pacientes sentados e em repouso por um tempo mínimo de cinco minutos; adotando-se valores de referência para a classificação de hipertensão arterial sistêmica de acordo com as VII Diretriz Brasileira de Hipertensão ${ }^{(20)}$.

Para o cálculo do índice de massa corpórea (IMC), realizaram-se medidas de peso e altura utilizando-se balança antropométrica mecânica calibrada (Welmy $\left.{ }^{\circledR}\right)$. Os valores de referência considerados para IMC em idosos estiveram de acordo com as Diretrizes Brasileiras de Obesidade de $2016^{(21)}$, aceitas pelo Ministério da Saúde.

As mensurações de circunferência abdominal (CA) foram realizadas com o abdome relaxado e fita métrica posicionada no ponto médio entre o rebordo costal inferior e a crista ilíaca, de acordo com recomendações da I Diretriz Brasileira de Diagnóstico e Tratamento da Síndrome Metabólica ${ }^{(22)}$. Definiu-se o ponto de corte para a CA, considerado como risco cardiovascular aumentado, conforme a I Diretriz de Prevenção Cardiovascular ${ }^{(7)}$, V Diretriz Brasileira de Dislipidemias e Prevenção da Aterosclerose ${ }^{(23)}$ e as Diretrizes Brasileiras de Obesidade de 2016(21).

Os idosos receberam guia para realização de exames de perfil lipídico, sendo orientados em relação aos procedimentos necessários para a realização dos mesmos, com recomendação de jejum de 12 horas, de acordo com protocolo do Ambulatório interno da Universidade. Para a classificação bioquímica dos exames de glicemia em jejum e perfil lipídico, utilizaram-se os parâmetros adotados pela V Diretriz Brasileira de Dislipidemias e Prevenção da Aterosclerose ${ }^{(23)}$. Para o levantamento a respeito de tabagismo, ocorrência de evento cardiovascular prévio e uso de medicações, foram aplicados questionários em forma de entrevista.

Ao término dos três meses de atividades, todos os idosos repetiram os exames laboratoriais de glicemia em jejum e perfil lipídico, e submetidos novamente à aferição de pressão arterial em repouso e medidas antropométricas, realizada pela mesma avaliadora nos dois momentos. A Tabela I representa os padrões de referência utilizados para os dados antropométricos. 
Tabela I - Padrões de referência utilizados para dados antropométricos. São Paulo, Brasil, 2017.

\begin{tabular}{lccc}
\hline Variáveis & Normal / Ideal (\%) & $\begin{array}{c}\text { Padrão de referência } \\
\text { Limítrofe (\%) }\end{array}$ & Alterado (\%) \\
\hline IMC $^{*}\left(\mathbf{K g} / \mathbf{m}^{\mathbf{2}}\right)$ & $>22 \mathrm{e}<27$ & & $\geq 27$ \\
Circunferência abdominal (cm) $_{\text {Colesterol total (mg/mL) }}$ & $<90 \mathrm{H},<80 \mathrm{M}$ & $200-239$ & $\geq 90 \mathrm{H}, \geq 80 \mathrm{M}$ \\
LDL-C** $(\mathbf{m g} / \mathbf{d L})$ & $<200$ & $130-159$ & $\geq 240$ \\
HDL-C & $<130$ & & $\geq 160$ \\
Triglicérides (mg/dL) & $>60$ & $150-199$ & $\geq 40$ \\
Glicemia de jejum (mg/dL) & $<150$ & $100-125$ & $\geq 126$ \\
Pressão arterial sistólica (mmHg) & $\leq 99$ & $121-139$ & $\geq 140$ \\
Pressão arterial diastólica (mmHg) & $\leq 120$ & $81-89$ & $\geq 90$ \\
\hline
\end{tabular}

H: Homem; M: Mulher; * Índice de Massa corpórea; ${ }^{* *}$ Lipoproteína de baixa densidade; ${ }^{* * *}$ Lipoproteína de alta densidade

Realizou-se o protocolo de exercícios entre os meses de abril e junho de 2016. Os idosos foram divididos em dois grupos para melhor organização das atividades, que aconteciam duas vezes por semana no período da tarde, dentro da Universidade Santo Amaro, Campus 1, com duração de aproximadamente noventa minutos por dia para cada grupo. Consistiu de trinta minutos de caminhada inicial, trinta minutos de exercícios ativos livres para membros superiores e tronco em forma de circuito, e trinta minutos de caminhada final. O circuito era composto por cinco movimentos realizados durante um minuto cada, repetindo-se seis séries: flexão de tronco, flexão de ombros, extensão de ombros, abdução horizontal de ombros, flexão lateral da coluna. A seleção desse protocolo baseou-se em dados da literatura acerca dos benefícios dos exercícios aeróbios, como as caminhadas, para redução da $\mathrm{HAS}^{(2-4)}$ e para controle dos demais fatores de risco cardiovascular, como obesidade, hiperlipidemia e hiperglicemia ${ }^{(14,15)}$. Para o circuito, considerou-se realizar exercícios livres com os membros superiores e tronco, pois não elevam tanto a frequência cardíaca, mas promovem alta estimulação da circulação periférica da musculatura envolvida ${ }^{(9,11,24-27)}$. O método de intercalar os movimentos, por meio de séries curtas de exercícios, consistiu em uma estratégia para motivar e incentivar o público idoso ${ }^{(18)}$. A ausência de equipamentos para incremento das atividades consistiu em uma limitação neste estudo.

Para análise dos dados, utilizou-se o programa SPSS, versão 20.0. O teste de Kolmogorov-Smirnov constatou que as variáveis avaliadas (peso, perfil lipídico, glicemia e pressão arterial) não apresentavam distribuição normal, portanto utilizou-se o teste de Wilcoxon para comparação dos valores obtidos na visita basal e final. Adotou-se nível de significância de $\mathrm{p}<0,05$.

O trabalho recebeu aprovação pelo Comitê de Ética em Pesquisa da Universidade Santo Amaro, Parecer nº 1436639, em consonância com o disposto na Declaração de Helsinki e na Resolução n 466/12 do Conselho Nacional de Saúde. Obteve-se também o seguinte Registro Brasileiro de Ensaios Clínicos: RBR-4nn8j5.

\section{RESULTADOS}

Dos 34 idosos que participaram da pesquisa, 25 eram do sexo feminino (74\%), e 47\% eram ex-tabagistas ( $\mathrm{n}=16)$. Além disso, idade mediana (intervalo interquartis) de 63 (61-66) anos. Os tabagistas corresponderam a 6\% (n=2) dos indivíduos e $53 \%(n=18)$ eram diabéticos. Com relação às medicações em uso, $88 \%(n=30)$ faziam uso de medicação anti-hiperglicemiante e $32 \%(\mathrm{n}=10)$ usavam estatinas; $15 \%(\mathrm{n}=5)$ tiveram pelo menos um desfecho (infarto agudo do miocárdio) nos últimos sete anos.

A Tabela II representa os dados obtidos antes e após o protocolo de exercícios para as variáveis peso, IMC, circunferência abdominal, colesterol total, LDL-C, HDL-C, triglicérides e glicemia de jejum. Houve redução significativa do peso e IMC, bem como da pressão arterial sistólica e diastólica $(\mathrm{p}<0,0001$ em todos os casos, teste de Wilcoxon).

\section{DISCUSSÃO}

O aumento da expectativa de vida no mundo, frequentemente acompanhado do desenvolvimento de $\mathrm{DCNT}^{(1)}$, tem direcionado ações para a promoção de um envelhecimento mais saudável por meio de programas de exercícios voltados à população idosa $^{(9,13,25-27)}$. Esses programas são estratégias de combate à HAS, podendo também ser determinantes para amenizar agravos relacionados à doença $\mathrm{a}^{(9,11,14,28)}$.

A amostra, predominantemente composta por indivíduos do sexo feminino (74\%), se justifica por um maior de mulheres terem aceitado participar do presente estudo, fato que pode ser justificado pela hipótese de os homens terem maior resistência para cuidarem da própria saúde; enquanto as mulheres preocupam-se com a aparência, peso e com o envelhecimento saudável. Pesquisas revelam que a maioria dos homens se considera imune às doenças, priorizando os prazeres da vida em detrimento à saúde(29)

Acredita-se que o alto número de ex-fumantes (47\%) encontrado na presente pesquisa deva-se às atuais campanhas de combate ao tabaco, como regulamentação da Lei Antifumo, aumento do preço do cigarro, proibição de propaganda de derivados 


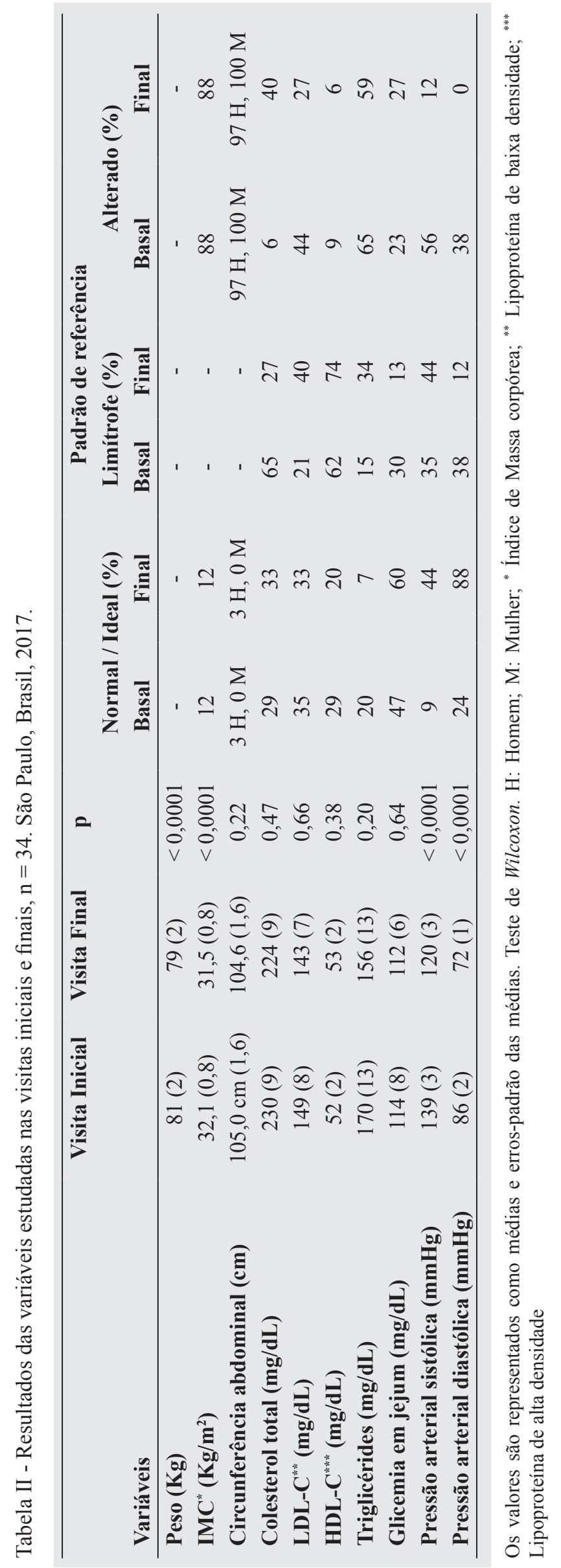


de fumo e extinção dos fumódromos na cidade de São Paulo. Pesquisa apresentada pelo Ministério da Saúde revela queda de 30,7\% no número de fumantes no Brasil nos últimos nove anos. A nicotina influi em uma série de doenças, destacando-se as doenças cardiovasculares. O hábito de fumar não se constitui num fator causador direto de HAS, mas pacientes hipertensos fumantes apresentam maior risco de morte por hipertensão maligna e por IAM ${ }^{(26)}$. Na amostra da atual pesquisa, $15 \%$ dos pacientes relataram ter sofrido IAM nos últimos sete anos, sendo este o motivo de $31 \%$ dos ex-fumantes terem parado de fumar; e nenhum relatou a ocorrência de AVE prévio.

Mais da metade $(53 \%, \mathrm{n}=18)$ dos participantes da atual pesquisa era diabético tipo 2, o que corrobora dados da literatura ${ }^{(1,2)}$, visto que a diabetes se constitui em um dos principais fatores de risco para as doenças cardiovasculares e para agravos de saúde tratados na rede de atenção básica.

Em relação às medidas de circunferência abdominal da amostra do presente estudo, pode-se verificar média acima dos valores aceitáveis para se prevenir o risco de agravos cardiovasculares ${ }^{(21)}$. E o ponto de corte para risco cardiovascular aumentado é definido conforme etnia e sexo, sendo igual ou superior a $90 \mathrm{~cm}$ para homens e $80 \mathrm{~cm}$ para mulheres em países sul-americanos ${ }^{(7,22,23)}$. O curto período de tempo de três meses em que foi realizado o protocolo de exercícios da presente pesquisa pode ter sido um fator determinante para não ter havido redução estatisticamente significante na CA, representada pela deposição excessiva de tecido adiposo abdominal visceral. Variáveis que não puderam ser controladas também devem ser consideradas, tais como o uso de outros medicamentos, além dos anti-hipertensivos, outras patologias, alimentação e condições socioeconômicas.

Os dados relativos ao peso corporal e ao IMC sugerem que a prática regular de exercícios físicos de intensidade leve a moderada tenha sido um fator de incentivo a hábitos de vida mais saudáveis para os idosos anteriormente inativos do atual estudo, contribuindo para redução estatisticamente significativa de peso corporal e, consequentemente, do IMC. O processo de envelhecimento ativo e a participação em um programa de exercícios físicos constitui uma medida que agudamente aumenta o gasto energético e parece contribuir para o combate à obesidade ${ }^{(14)}$. Sabe-se que pequenas mudanças nos hábitos diários representam requisitos essenciais para o tratamento da $\operatorname{HAS}^{(2,3)}$.

A dislipidemia representa um importante fator de risco de aterosclerose e DCV, uma vez que a gordura acumulada nas paredes das artérias pode levar à obstrução parcial ou total do fluxo sanguíneo que chega ao coração e ao encéfalo ${ }^{(30)}$. A característica da amostra avaliada na atual investigação em relação ao perfil lipídico foi de níveis limítrofes para todas as variáveis ${ }^{(23)}$. Entretanto, é importante considerar os demais fatores associados, como envelhecimento, obesidade, diabetes, IAM prévio e tabagismo ${ }^{(30,31)}$. Geralmente, a dislipidemia não manifesta sinais ou sintomas, mas estudos comprovam que esse distúrbio apresenta forte associação com obesidade abdominal e dieta inadequada rica em alimentos gordurosos, aumentando o risco carviovascular ${ }^{(30,31)}$.

De acordo com entrevista realizada no início do presente estudo, 32\% da amostra estava em uso de estatinas. Este dado sugere que pelo menos um terço dos pacientes sejam portadores de hiperlipidemia. Assim, acredita-se que o exercício físico, juntamente com a terapia medicamentosa, tenha contribuído para a manutenção da média desses valores em níveis limítrofes ${ }^{(23)}$. Também se encontrou redução na média dos níveis de colesterol total (CT), LDL-C e TG, pequena redução da glicemia de jejum e aumento de HDL-C, entretanto essas diferenças não foram estatisticamente significantes no estudo em questão. Possivelmente, o curto período de tempo do protocolo realizado e variáveis que não puderam ser controladas, como alimentação, medicamentos utilizados no tratamento de hiperlipidemia e outros tratamentos e hábitos de vida, tenham sido fatores limitantes, devendo também ser considerados.

Em um ensaio, realizado em 2008, foram observados 30 indivíduos, durante seis meses, divididos em dois grupos, e encontrouse redução significante em CT e elevação de HDL-C para o grupo que realizou exercício e dieta. O grupo que realizou apenas exercício apresentou significante redução de CT e LDL-C ${ }^{(32)}$. Esses resultados não corroboram os do presente estudo. Apesar da semelhança em relação ao tempo de protocolo e número de pacientes, no atual trabalho não foi oferecido um acompanhamento nutricional ao longo do período dos três meses, o que constitui uma limitação da pesquisa. Com relação à glicemia, os benefícios da atividade física são descritos na literatura, e é uma recomendação atual que sua prática seja regular para os indivíduos com sobrepeso e obesidade associada a diabetes, devendo fazer parte do tratamento ${ }^{(33)}$.

Os efeitos crônicos dos exercícios sobre a PA de indivíduos hipertensos tem sido objeto de vários estudos. Há um consenso na literatura de que o treinamento físico promova a diminuição da PA de repouso ${ }^{(7,20)}$. Entretanto, características específicas da prescrição, como o tempo de protocolo, intensidade e tipo de exercício, parecem exercer influência sobre o resultado das pesquisas. Reduções pressóricas foram observadas ao longo dos anos em estudos com protocolos que variam de quatro semanas a seis meses ${ }^{(9,10,15,32,34)}$.

Em 2004, verificou-se que o exercício físico regular, de intensidade moderada, três dias por semana, por 10 semanas, foi capaz de reduzir a PA de indivíduos com hipertensão moderada ${ }^{(35)}$. Porém, em 2006, outros autores encontraram que a diminuição da PA após seis meses de condicionamento físico foi significativamente maior do que a evidenciada após três meses ${ }^{(36)}$. Um outro trabalho, que envolveu 207 indivíduos, submeteu hipertensos a um programa de exercício físico por oito semanas. Os pacientes foram randomizados em cinco grupos, um grupo controle com sedentários e quatro grupos que diferiam em volume de exercícios semanais. Os autores verificaram que houve redução estatisticamente significativa tanto na PAS como na PAD em repouso nos quatro grupos submetidos ao treinamento, e que não houve redução maior com o aumento do volume de exercício. A magnitude de redução da PAD não foi significativamente diferente nos quatro grupos, demonstrando que o incremento de 
volume, ou seja, o total de minutos semanais, não foi um fator primordial para o efeito hipotensor dos exercícios ${ }^{(37)}$. Assim, as pesquisas sugerem que a prática regular de exercícios ao longo da vida parece possuir efeitos mais significativos na redução da PA do que o tempo total de minutos semanais realizados, colaborando para a saúde do idoso.

No presente estudo, observou-se que exercício físico de intensidade moderada, totalizando 180 minutos semanais, por um período de três meses, foi capaz de reduzir cronicamente e de maneira estatisticamente significativa tanto a PAS média quanto a PAD média de pacientes idosos com HAS moderada, comparando-se os valores iniciais, de quando os sujeitos se encontravam sedentários, com os valores finais, após três meses de atividades.

A busca de uma explicação para os efeitos crônicos do treinamento físico em pacientes hipertensos tem sido motivação de outros trabalhos ${ }^{(8,38)}$. Os mecanismos que envolvem a queda pressórica parecem estar relacionados a fatores tanto hemodinâmicos como humorais $^{(39)}$. Acredita-se que um dos efeitos crônicos do exercício de baixa intensidade seja a diminuição no débito cardíaco pela redução da frequência cardíaca de repouso ${ }^{(20)}$, em decorrência de menor estimulação simpática e aumento do número de impulsos transmitidos pelo nervo vago, gerando estimulação miocárdica parassimpática ${ }^{(39)}$.

Independentemente dos mecanismos subjacentes à ocorrência da hipotensão pós-exercício (HPE), pode-se sugerir que o exercício físico, mesmo que leve, mas realizado regularmente, seja uma estratégia não-farmacológica, clinicamente importante, para reduzir cronicamente os níveis de PAS e PAD da população idosa. Contudo, são necessárias mais investigações acerca dos fatores que influenciam essa redução e também estudos adicionais envolvendo exercício e idosos.

\section{CONCLUSÃO}

O efeito do programa de exercícios físicos de intensidade leve a moderada, realizado durante três meses, duas vezes por semana, foi eficaz na redução dos fatores de risco cardiovascular dos idosos sedentários e hipertensos estudados.

\section{CONFLITOS DE INTERESSE}

Os autores informam que não há conflitos de interesse no estudo realizado.

\section{REFERÊNCIAS}

1. Roth GA, Forouzanfar MH, Moran AE, Barber R, Nguyen G, Feigin VL, et al. Demographic and epidemiologic drivers of global cardiovascular mortality. N Engl J Med. 2015;372(14):1333-41.

2. Ranquinen T, Sarzynski MA, Ghoshi S, Bouchard C. Are there genetic paths common to obesity, cardiovascular disease outcomes, and cardiovascular risk factors? Circ Res. 2015;116(5):909-22.

3. Campos MA, Rodrigues JF, Silveira MF, Neves DMR, Vilhena JM, Oliveira JF, et al. Impacto dos fatores de risco para doenças crônicas não transmissíveis na qualidade de vida. Ciênc Saúde Colet. 2013;18(3):873-82.

4. Lavie CJ, Arena R, Swift DL, Johannsen NM, Sui X, Lee DC, et al. Exercise and the cardiovascular system: clinical science and cardiovascular outcomes. Circ Res. 2015;117(2):207-19.

5. Tan S, Li W, Wang J. Effects of six months of combined aerobic and resistance training for elderly patients with a long history of Type 2 Diabetes. Journal Sports Sci Med. 2012;11(3):495-501.

6. Moran AE, Odden MC, Thanataveerat PWR, Tzong KY, Rasmussen PW, Guzman D, et al. Cost-Effectiveness of Hypertension Therapy According to 2014 Guidelines. N Engl J Med. 2015;372(5):447-55.

7. Sociedade Brasileira de Cardiologia. I Diretriz Brasileira de Prevenção Cardiovascular. Arq Bras Cardiol. 2013;101(6 Suppl 2):1-63.

8. Anunciação PG, Polito MD. Hipotensão pós-exercício em indivíduos hipertensos: Revisão. Arq Bras Cardiol. 2011;96(5): 425-6.

9. Venturelli M, Cè E, Limonta E, Schena F, Caimi B, Carugo S, et al. Effects of endurance, circuit, and relaxing training on cardiovascular risk factors in hypertensive elderly patients. Age (Dordr). 2015;37(5):1-13.

10. Maruf FA, Salakob BL, Akinpelu AO. Can aerobic exercise complement antihypertensive drugs to achieve blood pressure control in individuals with essential hypertension? J Cardiovasc Med (Hagerstown). 2014;15(6):456-62.

11. Cavalcante PA, Rica RL, Evangelista A, Serra AJ, Figueira, Jr A, Pontes, Jr, et al. Effects of exercise intensity on postexercise hypotension after resistance training session in overweight hypertensive patients. Clin Interv Aging. 2015;10:1487-95.

12. Hall JE, Do Carmo JM, Da Silva AA, Wang Z, Hall ME. Obesity-induced hypertension: interaction of neurohumoral and renal mechanisms. Circ Res. 2015;116(6):991-1006. 
13. Silva BP, Santos LMN, Lima RSC, Cunha MAL. Efeito da prática da atividade física regular no risco cardiovascular em idosos hipertenso e/ou diabéticos: uma análise através do perfil lipídico. In: Congresso Internacional de Envelhecimento Humano. Campina Grande; 2013.

14. Kloster R, Liberali, R. Emagrecimento: composição corporal da dieta e exercício físico. Rev Bras Nutr Esporte. 2008;2(11):288306.

15. Silva AS, Zanesco A. Exercício físico, receptores B-adrenérgicos e resposta vascular. J Vasc Bras. 2010;9(2):47-56.

16. Campos GW, Barros RB, Castro AM. Avaliação de política nacional de promoção da saúde. Ciênc Saúde Colet. 2004;9(3):7459.

17. Tahan J. Reflexões de idosos participantes de grupos de promoção de saúde acerca do envelhecimento e da qualidade de vida. Saúde Soc. 2010;19(4):878-88.

18. Nicolau S, Mecenas AP, Freitas CMSM. Idosos praticantes de atividade física: perspectivas sobre o envelhecimento da população. Arq Ciências Saúde. 2015;22(2):69-74.

19. Dawalibi, NW, Anacleto GMC, Witter C, Goulart RMM, Aquino RC. Envelhecimento e qualidade de vida: análise da produção científica da SciELO. Estud Psicol. 2013;30(3):393-403.

20. Sociedade Brasileira de Cardiologia. VII Diretrizes Brasileiras de Hipertensão. Arq Bras Cardiol. 2016;107(3 Supl 3):1-83.

21. Associação Brasileira para o Estudo da Obesidade e da Síndrome Metabólica. Diretrizes brasileiras de obesidade 2016. $4^{\mathrm{a}}$ ed. São Paulo: ABESO; 2016.

22. Sociedade Brasileira de Cardiologia. I Diretriz Brasileira de Diagnóstico e Tratamento da Síndrome Metabólica. Arq. Bras. Cardiol. 2005;84(Supl 1):1-27.

23. Sociedade Brasileira de Cardiologia. V Diretriz Brasileira de Dislipidemias e Prevenção da Aterosclerose. Arq Bras Cardiol. 2013;101(4 Supl 1):1-221.

24. Carvalho RST, Pires CMR, Junqueira GC, Freitas D, Marchi-Alves LM. hypotensive response magnitude and duration in hypertensives: continuous and interval exercise. Arq Bras Cardiol. 2015;104(3):234-41.

25. Souto AL, Lima LM, Castro EA, Veras RP, Segheto W, Zanatta TC, et al. Blood pressure in hypertensive women after aerobics and hydrogymnastics sessions. Nutr Hosp. 2015;32(2):823-8.

26. Ammar T. Effects of aerobic exercise on blood pressure and lipids in overweight hypertensive postmenopausal women. J Exerc Rehabil. 2015;11(3):145-50.

27. Martelli A. Aspectos fisiopatológicos da aterosclerose e a atividade física regular como método não farmacológico no seu controle. Rev Saúde Desenvolvimento Humano. 2014;2(1):41-52.

28. Larsen MK, Matchkov VV. Hypertension and physical exercise: the role of oxidative stress. Medicina. 2016;52(1):19-27.

29. Araújo MG, Lima GAF, Holanda CSM, Carvalho JBL, Câmara AG. Saúde do homem: ações e serviços na estratégia da Saúde da Família. Rev Enferm UFPE. 2014;8(2):264-71.

30. Toussirot E, Michel F, Meneveau N. Exercice physique intense, atteinte musculaire et prise de statines. / Intense physical exercise, muscle involvement and statin use. Presse Med. 2014;43(6Pt 1):726-7.

31. Koozehchian MS, Nazem F, Kreider RB, Roberts WJ, Best TM, Rong Y, Zuo L. The role of exercise training on lipoprotein profiles in adolescent males. Lipids Health Dis. 2014;13(95):1-7.

32. Fagherazzi S, Dias RL, Bortolon F. Impacto do exercício físico isolado e combinado com dieta sobre os níveis séricos de HDL, LDL, colesterol total e triglicerídeos. Rev Bras Med Esp. 2008;14(4):381-6.

33. Wing RR, Bolin P, Brancati FL, Bray GA, Clark JM. Cardiovascular effects of intensive lifestyle intervention in Type 2 Diabetes. N Engl J Med. 2013;369(2):145-54.

34. Dekleva M, Lazic JS, Arandjelovic A, Mazic S. Beneficial and harmful effects of exercise in hypertensive patients: the role of oxidative stress. Hypertens Res. 2016; 40(1):15-20.

35. Tsai JC, Yang HY, Wang WH, Hsieh MH, Chen PT, Kao CC, et al. The beneficial effect of regular endurance exercise training on blood pressure and quality of life in patients with hypertension. Clin Exp Hypertens. 2004;26(3):255-65.

36. Nunes APOB, Rios ACS, Cunha GA, Baretto ACP, Negrão CE. Efeitos de um programa de exercício físico não-supervisionado e acompanhado a distância, via internet, sobre a pressão arterial e composição corporal em indivíduos normotensos e préhipertensos. Arq Bras Cardiol. 2006;86(4):289-96. 
37. Takata K, Ohita T, Tanaka H. How much exercise is required to reduce blood pressure in essential hypertensives: a doseresponse study. Am J Hypertens. 2003;16(8):629-33.

38. Halliwill JR, Buck TM, Lacewell AN, Romero AS. Postexercise hypotension and sustained postexercise vasodilatation: what happens after we exercise? Exp Physiol. 2013;98(1):7-18.

39. Bowles DK, Laughlin MH. Mechanism of beneficial effects of physical activity on atherosclerosis and coronary heart disease. J Appl Physiol. 2011;111(1):308-10.

\section{Endereço do primeiro autor:}

Marinella Nogueira da Silva Hortencio

Universidade de Santo Amaro - UNISA

Campus I

Rua Prof. Enéas de Siqueira Neto, 340

Bairro: Jardim das Imbuias

CEP: 04829-300 - São Paulo - SP - Brasil

E-mail: marinellahortencio@yahoo.com.br

\section{Endereço para correspondência:}

Carolina Nunes França

Universidade de Santo Amaro - UNISA

Campus I

Rua Prof. Enéas de Siqueira Neto, 340

Bairro: Jardim das Imbuias

CEP: 04829-300 - São Paulo - SP - Brasil

E-mail: carolufscar24@gmail.com 